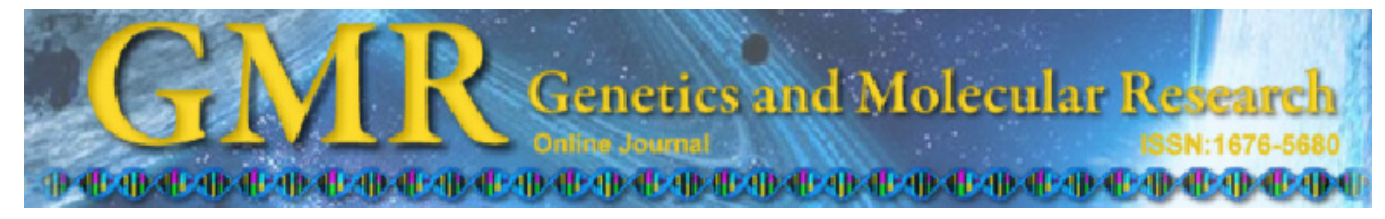

\title{
The effects of resveratrol on cyclooxygenase-1 and -2, nuclear factor kappa beta, matrix metalloproteinase-9, and sirtuin 1 mRNA expression in hearts of streptozotocin-induced diabetic rats
}

\author{
A.S. Yar, S. Menevse and E. Alp \\ Department of Medical Biology and Genetics, Faculty of Medicine, Gazi \\ University, Ankara, Turkey
}

Corresponding author: A.S. Yar

E-mail: atiyeseda@yahoo.com

Genet. Mol. Res. 10 (4): 2962-2975 (2011)

Received February 3, 2011

Accepted June 21, 2011

Published November 29, 2011

DOI http://dx.doi.org/10.4238/2011.November.29.7

\begin{abstract}
Resveratrol (RSV) has a beneficial role in the prevention of diabetes and alleviates some diabetic complications, such as cardiomyopathy. We investigated cyclooxygenase-1 (COX1), $\mathrm{COX}-2$, nuclear factor $\kappa \mathrm{B}(\mathrm{NF}-\mathrm{\kappa B})$, matrix metalloproteinase- 9 (MMP-9), and sirtuin 1 (SIRT1) mRNA expression levels in heart tissue after RSV treatment in streptozotocin (STZ)-induced diabetic rats. After induction of chronic diabetes with STZ, $10 \mathrm{mg} \mathrm{RSV} / \mathrm{kg}$ per day was administered to DM and DM+RSV groups for four weeks. At the end of the experiment, all rats were sacrificed and heart tissues were stored at $-80^{\circ} \mathrm{C}$; mRNA expression levels of COX-1, COX-2, NF- $\kappa$ B, MMP-9, and SIRT1 genes were analyzed with quantitative real-time PCR. We did not find any significant effect of RSV on
\end{abstract}


MMP-9, COX-1, COX-2, or NF-kB mRNA levels among the groups. However, SIRT1 mRNA levels decreased in the DM group compared to controls and increased in the DM+RSV group when compared to the DM group. SIRT1 is activated by RSV treatment in diabetic heart tissue. Activation of SIRT1 by RSV may lead to a new therapeutic approach for diabetic heart tissue. We conclude that RSV treatment can alleviate heart dysfunction by inhibiton of inflammatory gene expression such as SIRT1.

Key words: Diabetes mellitus; COX; NF-кB; SIRT1; Resveratrol; MMP-9

\section{INTRODUCTION}

The inflammation process plays an important role in pathogenesis of many diseases, such as diabetes mellitus (DM; Alberti and Zimmet, 1998). DM is a metabolic disease characterized by hyperglycemia resulting from defects of insulin secretion or insulin action or both (Alberti and Zimmet, 1998). There are two main forms of diabetes, type 1 and type 2 (Alberti and Zimmet, 1998). Both types are characterized by progressive $\beta$-cell failure and raised plasma glucose levels. Type $1 \mathrm{DM}$ is characterized by destruction of insulin-producing $\beta$-cells of Langerhans' islets in the pancreas leading to insulin deficiency. Type $1 \mathrm{DM}$ commonly occurs in children (Alberti and Zimmet, 1998). Type 2 DM is characterized by insulin resistance, reduced function of the pancreatic $\beta$-cells and relative insulin deficiency. It has been suggested that chronic hyperglycemia impairs $\beta$-cell function as a secondary adverse effect of diabetes. Type $2 \mathrm{DM}$ develops more frequently in adults than in children and it is the most common type of DM worldwide (Stumvoll et al., 2005). Diabetic cardiomyopathy is directly related to hyperglycemia that increases oxidative stress, inducing cardiac damage in animals and humans with diabetes (Alberti and Zimmet, 1998; Stumvoll et al., 2005; Robertson and Harmon, 2006).

Cyclooxygenase (COX) has two isoforms, COX-1 and COX-2, and is the key enzyme in arachidonic acid metabolism (Smith et al., 2000). Both COX isoforms metabolize arachidonic acid to generate prostaglandins (PG) and thromboxanes. Although both isoforms of COX share similar structural and biochemical properties, they are encoded on separate chromosomes and exhibit distinct physiological functions (Dubois et al., 1998; Smith et al., 2000).

COX-1 is a housekeeping enzyme, which is constitutively expressed in most tissues and thought to be involved in maintaining various physiological conditions, such as cytoprotection of gastric mucosa, regulation of renal blood flow, and control of platelet aggregation (Smith et al., 2000; Kotnik et al., 2005). In contrast, COX-2, the "inducible" isoform, is barely detectable in normal tissues but is responsible for the release of PGs during inflammatory conditions. For instance, DM and COX-2 are up-regulated by diverse stimuli including cytokines, mitogens, growth factors, and tumor promoters (Smith et al., 2000). The regulation of COX-2 transcription is mediated by several transcription factors such as

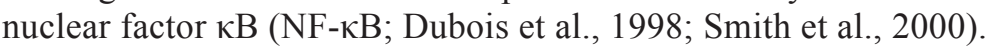


$\mathrm{NF}-\mathrm{\kappa B}$ is a nuclear transcription factor that regulates the expression of various genes involved in cell proliferation, adhesion, angiogenesis, apoptosis, cytoprotection, carcinogenesis, and inflammation (Karin, 1999). NF- $\mathrm{kB}$ consists of a 50-kDa subunit (p50) and a $65-\mathrm{kDa}$ subunit (p65). In unstimulated cells, NF- $\mathrm{kB}$ resides in the cytoplasm as an inactive complex with its inhibitory proteins, which are members of the IкB family. When the cellular

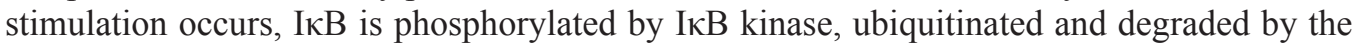

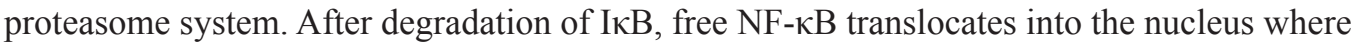
it regulates the expression of target genes involved in inflammation, cell proliferation, and apoptosis (Karin, 1999). Overexpression of NF- $\mathrm{kB}$ is also able to up-regulate the expression of metalloproteinase-9 (MMP-9; Philip et al., 2001). MMP-9, which is a zinc-dependent endopeptidase of a large MMP family, is related to tumor invasion and metastasis by its capacity for tissue remodeling via the extracellular matrix (St-Pierre et al., 2004).

Silencing 1nformation regulator 1 (Sirtuin 1 - SIRT1) is encoded by the SIRT1 gene, which is a member of the Sirtuin family (Milne et al., 2007; Jiang, 2008). SIRT1 is a deacetylase enzyme that regulates the activity of several transcriptional factors and enzymes responsible to nutrient availability, as well as a nicotinamide adenine dinucleotide (NAD+)-dependent histone deacetylase that regulates the metabolism and life span. Deacetylation of acetylated lysine residues on histone and non-histone substrate proteins is carried out by SIRT1. SIRT1, through its deacetylase activity, plays pivotal roles in various cellular processes, including genomic stability, transcriptional silencing, cell survival and longevity, and life span extension in response to calorie restriction (Rodgers et al., 2005; Milne et al., 2007). It is possible that high glucose decreases the cellular concentration of NAD, which is a cofactor for SIRT1. SIRT1 also stimulates glucose-dependent insulin secretion from pancreatic $\beta$-cells, involved in the processes of glucose homeostasis, and directly stimulates insulin signaling pathways in the pancreas (Lee et al., 2009). Some studies have shown that lower SIRT1 activity is reduced by high glucose levels in diabetic animal models and endothelial progenitor cells. Furthermore, it has been shown that overexpression of SIRT1 and several SIRT1 activators such as resveratrol (RSV) have beneficial effects on glucose homeostasis and insulin sensitivity in diabetic animal models (Milne et al., 2007; Jiang, 2008).

RSV is a natural polyphenolic compound and a naturally occurring phytoalexin present in a wide variety of plant species including mulberries, peanuts, nuts, and berries, and abundant in grapes (Fremont, 2000; Pervaiz, 2003). Grapes synthesize RSV in response to stress conditions such as fungal infection, UV irradiation, or exposure to ozone. RSV exists in both trans- and cis-isomeric forms. Since the trans-isomer is far more commonly found in plants, it has been extensively studied (Fremont, 2000; Martinez and Moreno, 2000; Pervaiz, 2003).

RSV has extensive biological activities, including antioxidant, anti-inflammatory, estrogenic, antiplatelet, anticarcinogenic, pro-apoptotic, and cardioprotective effects (Fremont, 2000; Martinez and Moreno, 2000; Pervaiz, 2003). The antioxidant activity of RSV may play an important role in its possible cardioprotective action. Although RSV is an inhibitor of COX1 at the enzyme level, it inhibits COX-2 both at gene expression and enzyme levels (Szewczuk

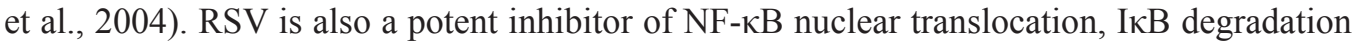
and NF-kB-dependent gene expression (Subbaramaiah et al., 1998; Kundu and Surh, 2004). RSV also suppresses MMP-9 expression by inhibiting NF- $\mathrm{kB}$ DNA binding activity (Yu et al., 2008). RSV, a potent activator of SIRT1, can improve the life span on cardiac function. Moreover, the most recent data suggest that RSV plays a beneficial role in the prevention of 
diabetes and alleviates some diabetic complications such as cardiomyopathy (Su et al., 2006). The reduction of SIRT1 activity due to high glucose levels correlates with the activation of MMP-9 transcription (Howitz et al., 2003).

RSV ameliorates common DM symptoms such as body weight loss, polyphagia, and polydipsia. RSV has also been shown to significantly decrease insulin secretion and delay the onset of insulin resistance in streptozotocin (STZ)-induced DM rats (Pervaiz, 2003; Su et al., 2006; Sharma et al., 2006).

The aim of the present study was to investigate the changes of COX-1, COX-2, NF-kB, SIRT1, and MMP-9 mRNA expressions after RSV treatment in STZ induced-diabetic rat hearts.

\section{MATERIAL AND METHODS}

\section{Drugs and chemicals}

STZ, RSV (3,5,4'-trihydroxy-trans-stilbene; approximately 99\% purity), dimethyl sulfoxide (DMSO), and sodium citrate buffer were obtained from Sigma (St. Louis, MO, USA). peqGOLD TriFast ${ }^{\mathrm{TM}}$ RNA isolation kit was purchased from Peqlab (Erlangen, Germany). Transcriptor First-Strand cDNA Synthesis kit, LightCycler TaqMan Master kit and RNase-Free DNaseI were purchased from Roche Diagnostics, GmbH (Mannheim, Germany). All other chemicals used in this experiment were of the highest purity.

\section{Animals}

Adult male Wistar rats (12 weeks old and 250-300 g body weight) were obtained from the Gazi University, Laboratory Animal Breeding and Experimental Research Center and all animal experimental protocols were performed in accordance with guidelines issued by the Local Institutional Committee for the Ethical Use of Animals at Gazi University (protocol No. G.U.ET- 06.073). The animals were maintained in individual polycarbonate cages at a constant temperature $\left(21^{\circ}\right.$ to $\left.24^{\circ} \mathrm{C}\right)$ and humidity of $30-40 \%$ on a 12 -h light/dark cycle. Animals were fed with standard diet and water ad libitum throughout the experiment. All animals were acclimatized for a minimum period of 1 week prior to the beginning of the study. Forty-four 3-month-old, Wistar albino male rats were used for the study as described previously (Yar et al., 2010). Animals were randomly divided into 6 experimental groups [group I: nondiabetic rats used as a control group $(\mathrm{N}=6)$; group II: sodium citrate buffer control group (sham control; $\mathrm{N}=7$ ); group III: STZ-induced diabetic group ( $\mathrm{DM} ; \mathrm{N}=9$ ); group IV: DMSO-induced control group (DMSO; $\mathrm{N}=6$ ); group $\mathrm{V}$ : RSV-treated sham control group (RSV; $\mathrm{N}=7$ ); group VI: RSV-treated diabetic group (DM+RSV; $\mathrm{N}=9)$ ].

\section{Induction of experimental diabetes}

Diabetes was induced according to the procedure described by Kumar et al. (2007). STZ was administered intraperitoneally (ip) at $55 \mathrm{mg} / \mathrm{kg}$ in a single dose and STZ was freshly prepared by dissolving in $0.1 \mathrm{M}$ sodium-citrate buffer, $\mathrm{pH}$ 4.5. Age- and weight-matched sham control rats received an equivalent amount of sodium-citrate buffer. Three days after STZ in- 
jection, tail-vein blood glucose levels were measured with a portable glucometer in all animals (Optium, Abbott, Santa Cruz, CA, USA). Rats with blood glucose concentrations $\geq 300 \mathrm{mg} /$ $\mathrm{dL}$ were considered as diabetic and used for the study. During the experiment, blood glucose levels were verified eight times (from the 3rd to the 60th day after the beginning of treatment) (Yar et al., 2010).

\section{Treatment with RSV}

Four weeks after diabetes induction, diabetic animals were divided into two groups, namely DM $(\mathrm{N}=9)$ and DM+RSV $(\mathrm{N}=9)$. Subsequently, animals, which belong to the RSV and DM+RSV groups, received the RSV injection, ip $(10 \mathrm{mg} / \mathrm{kg}$, per day) for 4 weeks (from the 5th week until the 8th week), while the age- and weight-matched DMSO group was treated with injection of equal volumes of DMSO $(10 \mathrm{mg} / \mathrm{kg}$, per day) given for 4 weeks (from the 5th week until the 8th week). RSV was administered according to the previous methods as described by Kumar et al. (2007) and Ates et al. (2007). RSV (10 mg/kg) was freshly prepared by dissolving in 30\% DMSO, diluted in $0.9 \% \mathrm{NaCl}$ and administered between 10 and $11 \mathrm{am}$ once a day for 30 days. At the end of the 8th week, all rats were sacrificed under ketamine hydrochloride and xylasin hydrochloride anesthesia. Afterwards, all hearts were removed, immediately frozen in liquid nitrogen and stored at $-80^{\circ} \mathrm{C}$ until analysis.

\section{RNA extraction and reverse transcription}

Total RNA was extracted from heart tissue (approximately $50-100 \mathrm{mg}$ ) by using the Trizol reagent. To avoid DNA contamination, we applied some modifications according to manufacturer instructions. RNA-containing pellet was treated with approximately 1-5 U RNase-free DNase per $\mu \mathrm{g}$ RNA and incubated at $37^{\circ} \mathrm{C}$ for $30 \mathrm{~min}$ before washing with $75 \%$ ethanol. The yield and quality of the RNA of each sample were determined by measuring the absorbance at 260 and $280 \mathrm{~nm}$ using the Nanodrop spectrophotometer (NanoDrop ND-1000, Montchanin, DE, USA). Total RNA ( $1 \mu \mathrm{g})$ was reverse transcribed in a $20-\mu \mathrm{L}$ reaction mixture using random hexamers and Transcriptor First-Strand cDNA Synthesis kit according to manufacturer instructions.

\section{Quantitative real-time PCR (RT-PCR) analysis}

COX-1, COX-2, NF- $\mathrm{BB}$ (p65), MMP-9, and SIRT1 mRNA expression levels were measured using RT-PCR with the LightCycler instrument. $\beta$-actin (ACTB) was used as a housekeeping gene in order to normalize COX-1, COX-2, NF-кB (p65), MMP-9, and SIRT1 expression levels. Probes and primers spanning exon-exon boundaries for each gene assay were designed using the Universal ProbeLibrary (UPL) Assay Design Center (http://www. roche-applied-science.com/sis/rtpcr/upl/index.jsp?id=UP030000). The primer and UPL probe numbers are presented in Table 1 . The reaction mixture prepared in glass capillaries contained $1 X$ LightCycler Taq-Man Master reaction mixture. Each sample was tested three times. PCR efficiency for each gene was determined by serial dilution method. Amplification efficiencies of the target genes and ACTB were approximately equal. Results were analyzed with the LightCycler Software version 3.5 (Roche Applied Science, GmbH, Mannheim, Germany) using the second derivate maximum method. 
Table 1. Gene-specific primer sequences and probe numbers.

\begin{tabular}{llll}
\hline Gene & Forward primer & Reverse primer & UPL probe No. \\
\hline ACTB & 5'CCCGCGAGTACAACCTTCT3' & 5'CGTCATCCATGGCGAACT3' & 17 \\
COX-1 & 5'GCTCCAGTTTCCCCCTGCT3' & 5'TTCTGGCATGGATAGTAACAACA3' & 77 \\
COX-2 & 5'ACCAACGCTGCCACAACT3' & 5'GGTTGGAACAGCAAGGATTT3' & 77 \\
NF- $B$ & 5'CTGGCAGCTCTTCTCAAAGC3' & 5'CCAGGTCATAGAGAGGCTCAA3' & 25 \\
SIRT1 & 5'GAAAATGCTGGCCTAATAGACTTG3' & 5'TGGTACAAACAAGTATTGATTACCG3' & 68 \\
MMP-9 & 5'CCTCTGCATGAAGACGACATAA3' & 5'GGTCAGGTTTAGAGCCACGA3' & 42 \\
\hline
\end{tabular}

\section{Statistical analysis}

Differences in body weight and blood glucose levels were performed by using the nonparametric Kruskal-Wallis test and the Mann-Whitney U-test. P values lower than 0.05 were considered to be significant. All statistics were analyzed using the SPSS statistical software (version 15.0 for Windows; SPSS Inc., Chicago, IL, USA). We performed a one-way ANOVA to assess differences between onset and end body weight and blood glucose levels. Statistical significance of differences in mRNA expression was analyzed by the pairwise fixed reallocation randomization test as statistical model included in the REST $^{\circ}$ software (Relative Expression Software Tool) developed for group-wise comparison and statistical analysis of relative expression results.

\section{RESULTS}

\section{Effects of RSV on plasma glucose levels and body weight}

The body weight and blood glucose levels determined at the end of the experiment are presented in Figure 1. At the onset of the study all animals had similar body weights. The body weights of the diabetic rats (264.9 \pm 16.73 for group III and $244.6 \pm 9.36$ for group VI) were found to be significantly lower than those of the age-matched control rats at the end of the study $(338 \pm 10.47$ for control, $347.4 \pm 5.35$ for sham control, $315.2 \pm 2.28$ for DMSO group, 309.3 \pm 6.49 for RSV group) $(\mathrm{P}<0.05)$. After RSV treatment, there was no significant difference between the body weights of diabetic and RSV-treated diabetic rats $(\mathrm{P}>0.05)$ (Figure 1A).
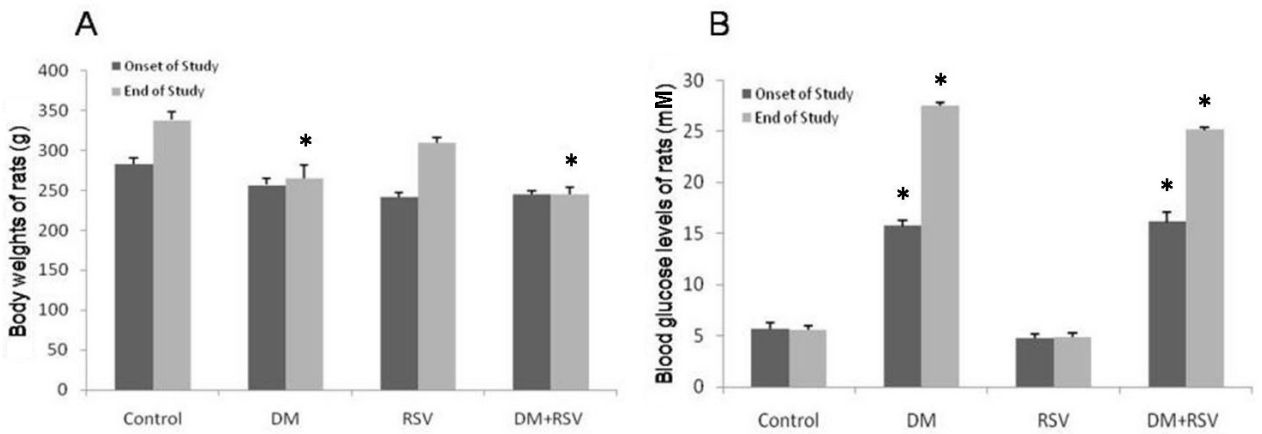

Figure 1. A. Body weights of each group at the beginning (after developing DM) and at the end of the study. B. Blood glucose levels of each group at the beginning and at the end of the study. Control group ( $N=6$ ), diabetic group (DM, $N$ $=9)$, resveratrol-treated group (RSV, $N=7), \mathrm{RSV}$-treated diabetic group $(\mathrm{DM}+\mathrm{RSV}, \mathrm{N}=9)$. Data are reported as means \pm SEM. *Significant from control and RSV groups $(\mathrm{P}<0.05)$. 
Blood glucose levels of diabetic rats were significantly higher than the nondiabetic groups at the beginning of the experiment $(\mathrm{P}<0.05)$. At the end, treatment with RSV did not affect blood glucose levels in diabetic rats $(27.62 \pm 0.23 \mathrm{mM})$ compared to the untreated diabetic group $(27.52 \pm 0.16 \mathrm{mM})$, both of which were significantly higher than the control and sham control groups $(5.54 \pm 0.16$ and $4.97 \pm 0.11 \mathrm{mM}$, respectively) $(\mathrm{P}<0.01)$. Blood glucose levels were slightly reduced in RSV-treated diabetic rats compared to the untreated diabetic groups but this reduction was not statistically significant $(\mathrm{P}>0.05)$ (Figure $1 \mathrm{~B})$.

\section{Effects of RSV on mRNA expression of COX-1, COX-2, NF-кB (p65), and MMP-9}

RT-PCR was performed in the STZ-induced DM rat model to investigate the role of RSV in the regulation of COX-1, COX-2, NF- $\mathrm{kB}$ (p65), and MMP-9 mRNA expressions in heart tissue. The mRNA expressions of COX-1, COX-2, NF- $\mathrm{KB}$ (p65), and MMP-9 were changed among the different treatment groups. mRNA levels of COX-1 and MMP-9 did not show any significant alteration in the study groups compared to the control group $(\mathrm{P}>0.05)$ (Figure 2).

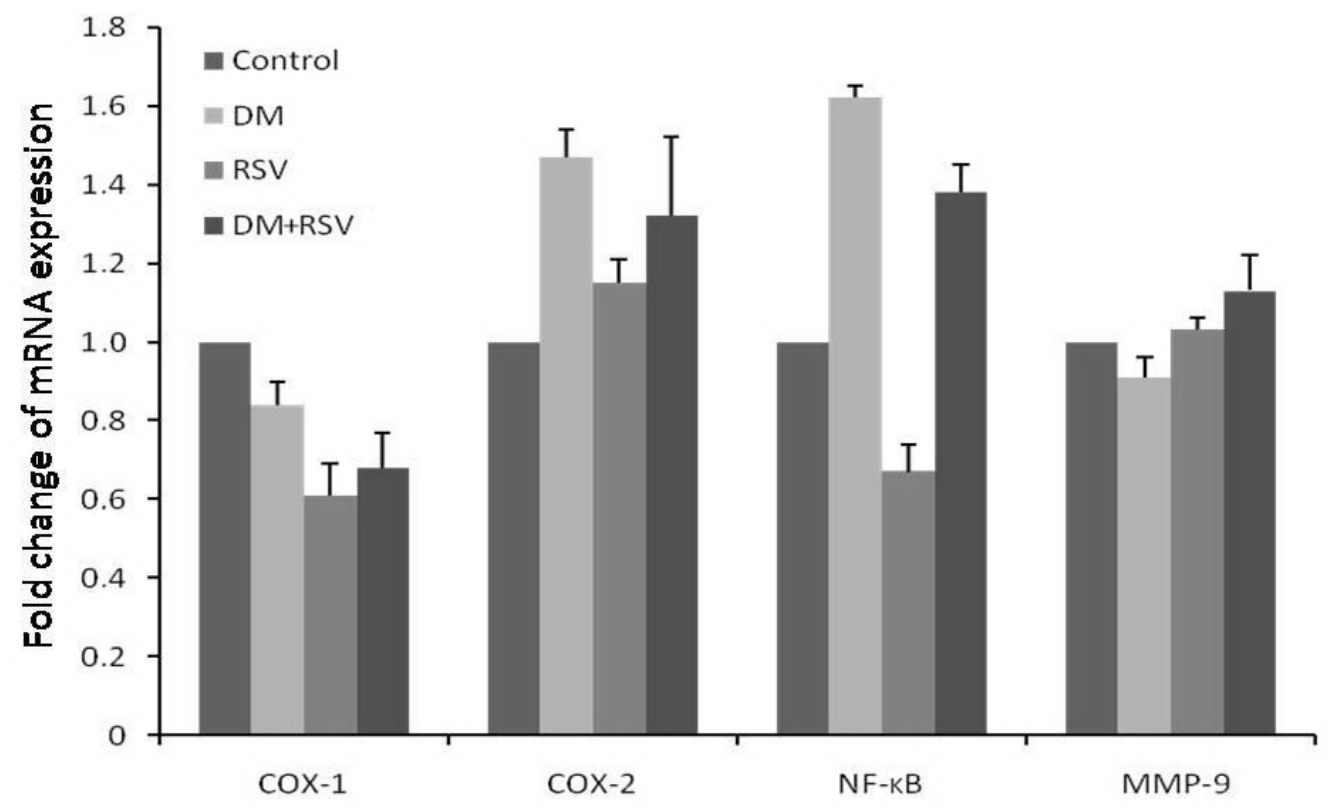

Figure 2. Relative mRNA expressions of COX-1, COX-2, NF- $\mathrm{B}$, and MMP-9. Bars represent mRNA expression normalized to $\beta$-actin and relative to control animals using the REST. For abbreviations, see legend to Figure 1.

mRNA levels of COX-2 and NF- $\mathrm{BB}(\mathrm{p} 65)$ increased 1.47- and 1.62-fold in the DM group compared to the control group, respectively (Figures 2 and 3). However, there was no statistically significant difference between the two groups $(\mathrm{P}>0.05)$ (Table 2). Comparing RSV and control groups, a 1.15-fold increased COX-2 mRNA level and a 1.49-fold decreased $\mathrm{NF}-\mathrm{kB}$ (p65) mRNA expression were seen in the RSV-treated group but the difference was not 
statistically significant $(\mathrm{P}>0.05)$ (Figure 2, Table 1). RSV-treated DM rats also had 1.32- and 1.38-fold increased mRNA levels of COX-2 and NF- $\kappa \mathrm{B}$ (p65) compared to the control group, respectively $(\mathrm{P}>0.05)$. Comparing $\mathrm{DM}$ and $\mathrm{DM}+\mathrm{RSV}$ groups, approximately a 1.2 -fold decrease in COX-2 and NF- $\mathrm{KB}$ (p65) mRNA expressions was seen in the DM+RSV group. However, there was no statistically significant alteration in COX-2 and NF- $\kappa B$ (p65) mRNA levels between the two groups $(\mathrm{P}>0.05)$.

Table 2. Statistical analysis results (P values) of REST (Relative Expression Software Tool) comparing different animal-treated groups.

\begin{tabular}{|c|c|c|c|c|c|}
\hline Groups & COX-1 & $\mathrm{COX}-2$ & $N F-\kappa B$ & SIRT1 & MMP-9 \\
\hline Control and sham control & 0.448 & 0.632 & 0.318 & 0.836 & 0.911 \\
\hline Control and DM & 0.848 & 0.376 & 0.136 & 0.023 & 0.731 \\
\hline Control and DMSO & 0.679 & 0.756 & 0.221 & 0.654 & 0.547 \\
\hline Control and RSV-treated sham control & 0.208 & 0.924 & 0.898 & 0.002 & 0.754 \\
\hline Control and RSV-treated DM & 0.745 & 0.453 & 0.492 & 0.646 & 0.673 \\
\hline Diabetes and RSV-treated DM & 0.834 & 0.679 & 0.458 & 0.006 & 0.360 \\
\hline
\end{tabular}

${ }^{a}$ COX-1 and COX-2 mRNA expression normalized to $\beta$-actin and relative to control animals using REST. DM = diabetes mellitus; $\mathrm{RSV}=$ resveratrol.

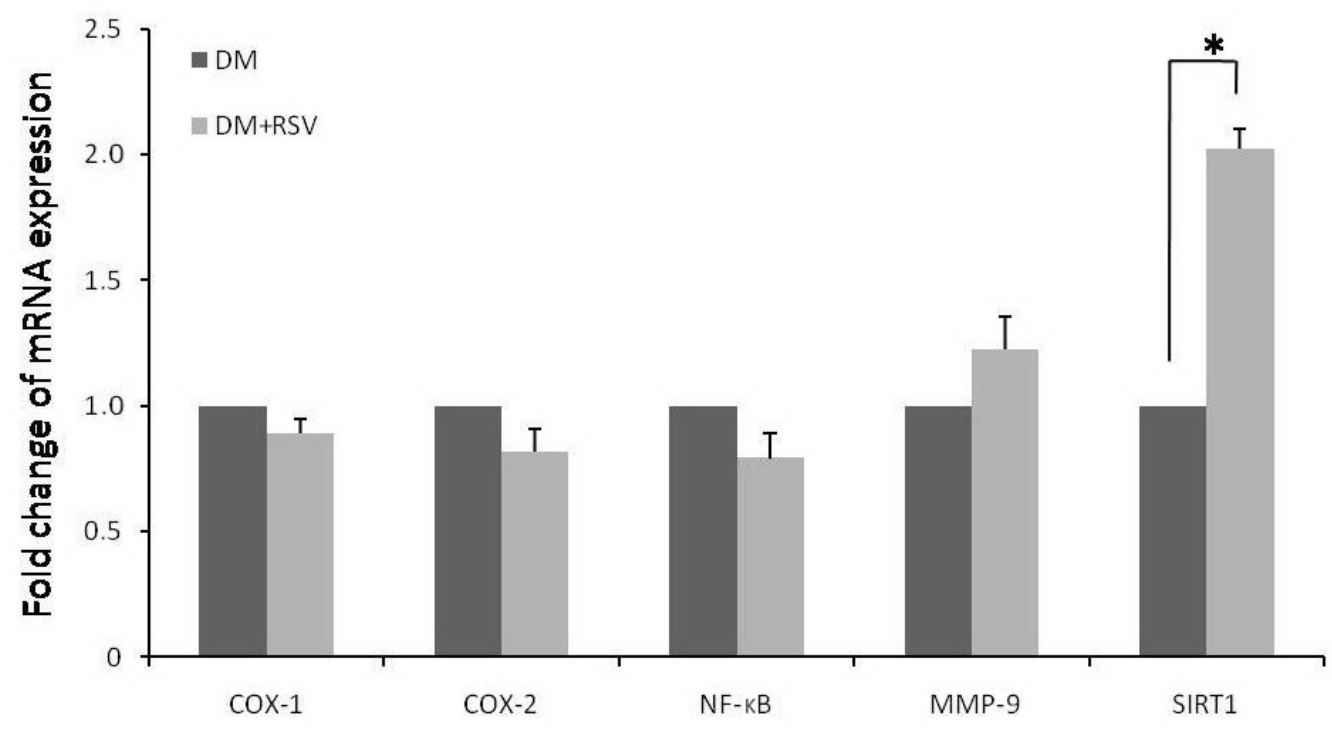

Figure 3. Relative mRNA expression of COX-1, COX-2, NF- $\mathrm{B}$, MMP-9, and SIRT1 compared between DM and $\mathrm{DM}+\mathrm{RSV}$. Bars represent mRNA expression normalized to $\beta$-actin and relative to control animals using the REST. $* \mathrm{P}<0.05$. For abbreviations, see legend to Figure 1 .

\section{Effects of RSV on SIRT1 mRNA expression}

To investigate the role of RSV in the regulation of SIRT1 mRNA expression in heart tissue, RT-PCR was performed in an STZ-induced DM rat model. SIRT1 mRNA levels were found to be decreased in the diabetic group $(\mathrm{P}<0.005)$. In addition, SIRT1 mRNA expression 
was increased in the RSV-treated group $(\mathrm{P}<0.05)$. However, the SIRT1 mRNA levels of the $\mathrm{DM}+\mathrm{RSV}$ group were higher than those of the untreated diabetic group $(\mathrm{P}<0.05)$ (Figure 4, Table 2). RT-PCR showed that mRNA expression of SIRT1 decreased 1.75-fold in the DM group compared to the control group $(\mathrm{P}<0.05)$. Comparing RSV-treated DM and control groups, a 1.95-fold increase in SIRT1 mRNA expression was seen in the RSV-treated DM group $(\mathrm{P}<0.05)$ (Figure 4, Table 2). RSV-treated DM rats had also approximately a 2-fold higher SIRT1 mRNA expression compared to untreated DM animals.

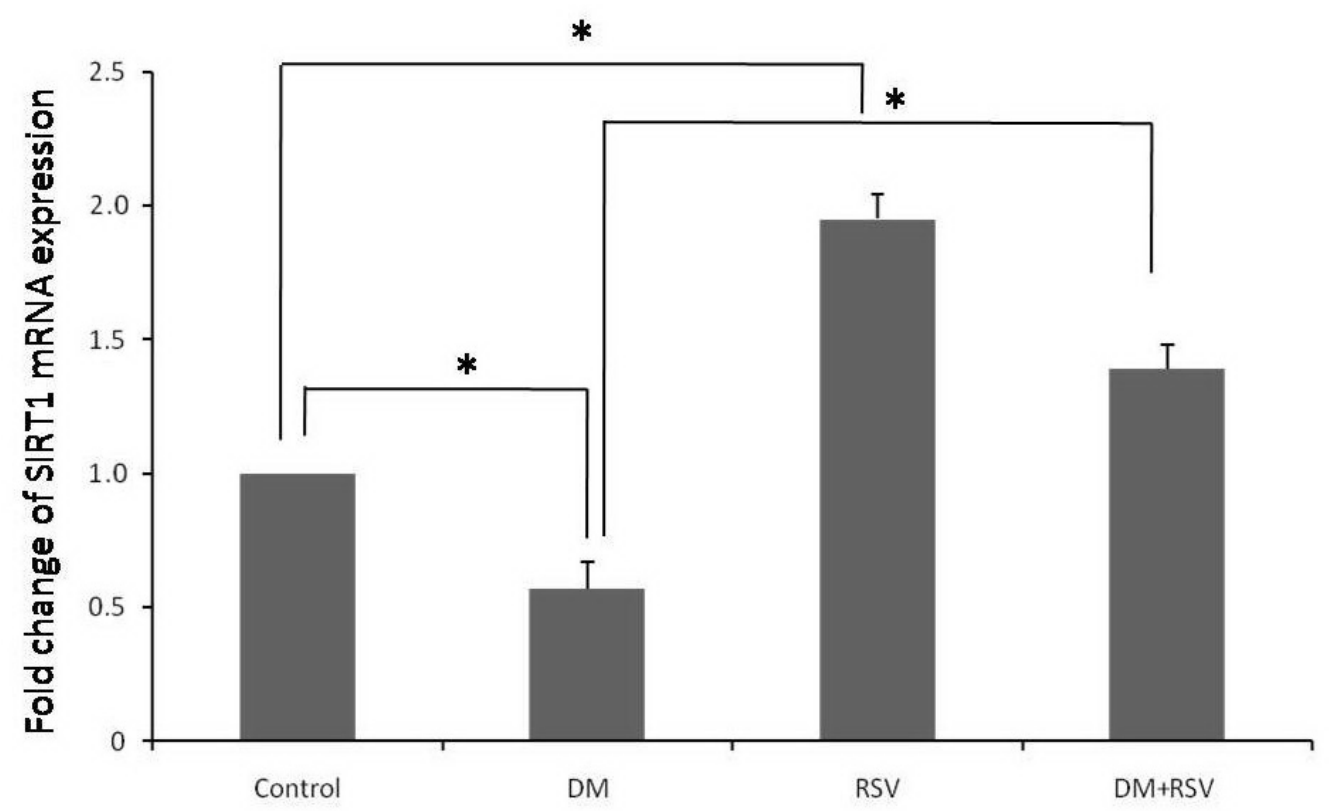

Figure 4. Relative mRNA expression of SIRT1. Bars represent mRNA expression normalized to $\beta$-actin and relative to control animals using the REST. $* \mathrm{P}<0.05$. For abbreviations, see legend to Figure 1.

\section{DISCUSSION}

The biological activities of RSV have been reported for at least 20 years. RSV has been reported to have a wide variety of biological effects as anti-oxidant, anti-cancer and antiinflammatory activities via down-regulation of COX-2 through suppression of NF-kB activation (Fremont, 2000; Pervaiz, 2003). In therapy-based studies, the effects of RSV on different biological systems were investigated in vivo and in vitro (Martinez and Moreno, 2000; Yu et al., 2002; Pervaiz, 2003). However, little is known about its effect on COX-1, COX-2, NF-кB, MMP-9, and SIRT1 mRNA expression levels in STZ-induced diabetic rat hearts.

In several in vivo studies, RSV showed selective COX-1 inhibition in doses of 5-10 mg.kg-1. day ${ }^{-1}$ (Bertelli et al., 1998; Juan et al., 2002). RSV doses generally range between 10 and $20 \mathrm{mg} / \mathrm{kg}$ in the literature (Bertelli et al., 1998; Juan et al., 2002; Pervaiz, 2003). In our study, RSV dose was $10 \mathrm{mg} \cdot \mathrm{kg}^{-1}$. day ${ }^{-1}$, which was within the limits of other studies investigating biological effects of RSV (Bertelli et al., 1998; Andlauer et al., 2000; Juan et al., 2002). 
The most recent data suggest that RSV plays a beneficial role in the prevention of diabetes and alleviates some diabetic complications, including cardiomyopathy, nephropathy, neuropathy, and retinopathy (Fremont, 2000; Pervaiz, 2003). Su et al. (2006) also observed that RSV reduces the common DM symptoms such as body weight loss, polyphagia and polydipsia in STZ-induced diabetic rats. RSV treatment significantly reduced the plasma glucose level in STZ-treated animals (Su et al., 2006; Sharma et al., 2006). The exact mechanism of RSV-induced amelioration of diabetic state remains unknown. We investigated the effects of RSV on body weight, blood glucose level and diabetic complications. At the onset of the study, all animals had similar body weights. After STZ injection, body weights of the diabetic rats were significantly lower than the age-matched control rats $(\mathrm{P}<0.05)$. However, any preventive effect of RSV on weight loss was not observed. On the other hand, there was no statistically significant reduction in blood glucose levels in the DM+RSV group compared to the DM group $(\mathrm{P}>0.05)$. Thus, treatment with RSV did not show any significant effect on body weight and blood glucose levels of rats with DM during the experimental period (Figure $1 \mathrm{~A}$ and $\mathrm{B})(\mathrm{P}>0.05)$. Based on these data, RSV at high concentrations or long-term therapy may be more effective than the current therapeutic strategy.

Numerous natural and artificial chemical substances such as antioxidants and antiinflammatory drugs have been studied to reduce the inflammatory effects of COX (Dubois et al., 1998; Smith et al., 2000). RSV, a potent antioxidant, was also investigated because of its anti-inflammatory feature. According to some studies, protein and gene expression levels of COX were inhibited by RSV (Subbaramaiah et al., 1998; Fremont, 2000; Martinez and Moreno, 2000; Pervaiz, 2003; Kundu and Surh, 2004). Subbaramaiah et al. (1998) found that RSV suppressed the synthesis of PGs by the inhibition of COX-2, without COX-1. They indicated that RSV inhibits COX-2 enzyme activity by suppressing COX-2 gene transcription without altering the amount of COX-1. COX-2, the inducible form, is expressed during inflammation process and is induced by proinflammatory and mitogenic stimuli (Dubois et al., 1998; Smith et al., 2000). Furthermore, Kundu and Moreno (2004) observed that RSV inhibits COX-2 mRNA and protein expressions at the tumor growth on rat skin. Martinez and Moreno (2000) demonstrated that RSV treatment inhibits protein expression of COX-2 and suppresses inflammatory PG production without influencing COX-1. The effect of RSV on the protein expression of COX-2 was also investigated by Banerjee et al. (2002) in an experimental breast cancer rat model. They indicated that RSV inhibits COX-2 protein and the expression of COX-2 mRNA via suppressing the NF- $\mathrm{kB}$ transcription factor. Thus, harmful effects of DM were reduced by RSV through inhibition of both COX-2 pathway and $\mathrm{PGE}_{2}$ synthesis (Subbaramaiah et al., 1998; Martinez and Moreno, 2000; Pervaiz, 2003; Szewczuk et al., 2004). In contrast, Jang et al. (1997) found that RSV could selectively inhibit COX activity by inhibiting COX-1 pathway, but not through COX-2 pathway and Szewczuk et al. (2004) confirmed this finding. Although RSV is thought to be a specific inhibitor of COX-1 (Szewczuk et al., 2004), at high concentrations it has been reported to reduce basal COX-2 activity in vitro (Jang et al., 1997). Our previous study demonstrated that RSV reduced COX-2 and especially COX-1 mRNA level but there were no statistically significant differences between DM and DM+RSV groups in STZ-induced DM rat kidney tissue (P > 0.05) (Yar et al., 2010). Similarly, RSV reduced the COX-1 mRNA level in STZ-induced DM rat heart tissues in the present study. However, this decline of COX-1 mRNA level was not statistically significant. In addition, there was no statistically significant decrease in the level of COX-2 mRNA. Thus, in our study, RSV did 
not show a meaningful effect on COX-1 and COX-2 mRNA expressions in DM+RSV group heart tissues. As in Jang et al. (1997) and Szewczuk et al. (2004), our results suggest that RSV could inhibit COX-2 mRNA expression at a high concentration. Therefore, high concentration of RSV may be appropriate to prevent COX-2-mediated inflammation in the STZ-induced diabetic rat heart tissue.

$\mathrm{NF}-\mathrm{\kappa B}$ controls the transcription of a variety of genes, including tumor-promoting COX-2, inducible nitric oxide synthase (iNOS), MMP-9, and endothelial adhesion molecules (Surh et al., 2001; Banerjee et al., 2002). The expression of these genes was reported to be down-regulated by RSV in different cell lines (Tsai et al., 1999; Asou et al., 2002). It has also been reported that RSV is a potent inhibitor of NF- $\mathrm{KB}$ activation and NF- $\mathrm{KB}$ signaling pathways by decreasing expression of various genes associated with inflammation and oxidative stress (Surh et al., 2001; Kundu and Surh, 2004). Its inhibition possibly occurs by promoting deacetylation of RelA/p65 protein at lysine 310 (Lys310) transcription (Holmes-McNary and Baldwin Jr., 2000; Manna et al., 2000). Kundu and Surh (2004) demonstrated that RSV pretreatment inhibited DNA binding of NF- $\mathrm{kB}$ in TPA-stimulated mouse skin. Our results are in contrast with various results reported by Kundu and Surh (2004) and Surh et al. (2001) in which RSV was a potent inhibitor of NF-KB (p65) activation. In the present study, we have shown that NF- $\mathrm{kB}$ (p65) mRNA levels were decreased in RSV and DM+RSV groups compared with control and diabetic groups, respectively. However, we could not demonstrate a statistically significant depletion in NF- $\mathrm{kB}$ (p65) mRNA expression. Therefore, RSV showed insufficent inhibition in NF- $\kappa B$ (p65) mRNA levels in STZ-induced diabetic rat heart tissue (P $>0.05$ ). It is known that activation of NF- $\mathrm{kB}$ is responsible for the COX-2 upregulation and contributed to the inflammatory process. In contrast, inhibition of NF- $\mathrm{KB}$ activation by the antioxidant chemical RSV inhibited COX-2 expression and alleviated inflammation. Based on this information, our results for COX-2 and NF- $\kappa \mathrm{B}$ mRNA expression showed similarities in diabetic rat heart tissue. Therefore, COX-2 expression is induced in parallel with NF- $\mathrm{KB}$ mRNA expression in the inflammatory process of DM. As in COX-2 mRNA expression, there was not a statistically significant inhibition of NF- $\mathrm{KB}$ mRNA expression. It is possible that our selected RSV concentration could be inadequate to effectively suppress COX-2 and NF- $\mathrm{KB}$ mRNA expression in the STZ-induced diabetic rat heart tissue.

SIRT1 has significant regulatory effects on the insulin mechanism. Moreover, SIRT1mediated inhibition of NF- $\mathrm{KB}$ causes multiple downstream effects of cytokine signaling pathway and may have beneficial effects in both cardiovascular and metabolic diseases (Milne et al., 2007; Pillarisetti, 2008; Jiang, 2008; Pfluger et al., 2008). Cardiac-specific overexpression of SIRT1 shows protection against oxidative stress in heart tissue (Alcendor et al., 2007).

SIRT1 activators, especially RSV, have been thoroughly validated in many preclinical models of metabolic and cardiovascular diseases (Pillarisetti, 2008). RSV is the first SIRT1 activator that has been suggested to activate the enzyme activity of SIRT1 both in vitro and in vivo (Howitz et al., 2003; Wood et al., 2004; Borra et al., 2005). Howitz et al. (2003) reported that RSV is a potent pharmacological agonist of SIRT1 activity through an allosteric interaction. Because aging leads to a progressive decline in multiple organ systems, it is not surprising that the importance of SIRT1, which has positive effects on several age-related disorders, is now being understood in diabetes and cardiovascular diseases (Milne et al., 2007; Pillarisetti, 2008; Pfluger et al., 2008). Under physiological conditions, SIRT1 activity is increased by fasting and reduced by high level of glucose (Nemoto et al., 2004). In addition, activation 
of SIRT1 by RSV may inhibit transcriptional activation of MMP-9 by targeting c-JUN (Woo et al., 2004; Yu et al., 2008). Moreover, Gao and Ye (2008) reported that a reduction in SIRT1 may be involved in increased AP-1 activity and MMP-9 expression in diabetic patients with hyperglycemia. Furthermore, Woo et al. (2004) showed that RSV inhibited MMP-9 mRNA expression by a mechanism involving NF-kB and AP-1. However, our data suggest that RSV did not have a significant effect on MMP-9 mRNA levels in STZ-induced diabetic rat heart tissue. When DM and DM+RSV groups were compared, the difference in MMP-9 gene expression was not statistically significant $(\mathrm{P}>0.05)$.

Cardiac-specific overexpression of SIRT1 prevents cardiac hypertrophy, apoptosis, cardiac dysfunction, and expression of senescence markers, which increases with age (Borra et al., 2005; Alcendor et al., 2007). Sulaiman et al. (2010) demonstrated that the enzymatic activity of cardiac SIRT1 is reduced in chronic diabetes and the activation of SIRT1 by RSV enhances expression of sarcoplasmic calcium ATPase and improves cardiac function. As reported by Alcendor et al. (2007), it was interesting to find that a moderate increase in the expression of SIRT1 had a cardioprotective effect, whereas a more severe overexpression of SIRT1 induced cardiomyopathy in an SIRT1 transgenic mice study. Thus, a careful evaluation regarding the dosage may be necessary in order to benefit from SIRT1 therapeutic potential. Moderate in vivo SIRT1 activators (2-4-fold activation) may be ideal rather than potent activators (10-fold or greater) to realize all the benefits of SIRT1 without side effects (Pillarisetti, 2008). Tanno et al. (2010) showed that Mn-SOD induced by RSV via nuclear SIRT1 reduced oxidative stress and participated in cardiomyocyte protection and preserved cardiac function. In the present study, SIRT1 mRNA levels decreased in the diabetic group $(\mathrm{P}<0.05)$, although it was increased in the DM+RSV group when compared to the control group $(\mathrm{P}<0.05)$. Therefore, SIRT1 is activated by RSV treatment in diabetic heart tissue and its mRNA expression was stimulated in the DM+RSV group compared to the DM group. According to our results, the activation effects of RSV on SIRT1 mRNA levels appeared to be compatible with the studies reported in literature (Howitz et al., 2003; Wood et al., 2004; Borra et al., 2005). SIRT1 was activated by RSV treatment in diabetic heart tissue and its mRNA expression was stimulated in the DM+RSV group compared to the DM group $(\mathrm{P}<0.05)$. Activation of SIRT1 by RSV may prevent proinflammatory phenotypic alterations in heart tissue. The activation of SIRT1 by RSV may be a promising therapeutic approach for insulin resistance and for treating DM-related complications such as cardiomyopathy. Our study indicates that RSV-induced SIRT1 protects heart tissue against hyperglycemia, which increases heart disease risk.

In conclusion, the present study demonstrates that intraperitoneal administration of RSV in diabetic rats may protect the heart against inflammation associated with DM via activated SIRT1. It is considered that high dose of RSV may reduce diabetic symptoms or ameliorate secondary complications of diabetes and alter mRNA levels of COX-1, COX-2, NF- $\mathrm{kB}$ (p65), and MMP-9, which play an important role in inflammation process. RSV, likely via an SIRT1-dependent mechanism, abrogated the adverse effects of hyperglycemia and prevented proinflammatory phenotypic alterations in heart tissue. Further studies are needed to define the mechanisms by which SIRT1 activation by RSV reduces chronic diabetic complications. Furthermore, additional studies are required to define the time range of treatment whereby RSV promotes cardioprotection after administration in higher concentrations. Thus, we presume that the cardioprotective effect of RSV at least partly contributes to its ability to stimulate SIRT1 synthesis in diabetic heart tissue. 


\title{
ACKNOWLEDGMENTS
}

\author{
Research supported by Gazi University Research Fund (project \#01/2007-34).
}

\section{REFERENCES}

Alberti KG and Zimmet PZ (1998). Definition, diagnosis and classification of diabetes mellitus and its complications. Part 1: diagnosis and classification of diabetes mellitus provisional report of a WHO consultation. Diabet. Med. 15: 539-553.

Alcendor RR, Gao S, Zhai P, Zablocki D, et al. (2007). Sirt1 regulates aging and resistance to oxidative stress in the heart. Circ. Res. 100: 1512-1521.

Andlauer W, Kolb J, Siebert K and Furst P (2000). Assessment of resveratrol bioavailability in the perfused small intestine of the rat. Drugs Exp. Clin. Res. 26: 47-55.

Asou H, Koshizuka K, Kyo T, Takata N, et al. (2002). Resveratrol, a natural product derived from grapes, is a new inducer of differentiation in human myeloid leukemias. Int. J. Hematol. 75: 528-533.

Ates O, Cayli S, Yucel N and Altinoz E (2007). Central nervous system protection by resveratrol in streptozotocin-induced diabetic rats. J. Clin. Neurosci. 14: 256-260.

Banerjee S, Bueso-Ramos C and Aggarwal BB (2002). Suppression of 7,12-dimethylbenz(a)anthracene-induced mammary carcinogenesis in rats by resveratrol: role of nuclear factor-kappaB, cyclooxygenase 2, and matrix metalloprotease 9. Cancer Res. 62: 4945-4954.

Bertelli AA, Giovannini L, Stradi R, Urien S, et al. (1998). Evaluation of kinetic parameters of natural phytoalexin in resveratrol orally administered in wine to rats. Drugs Exp. Clin. Res. 24: 51-55.

Borra MT, Smith BC and Denu JM (2005). Mechanism of human SIRT1 activation by resveratrol. J. Biol. Chem. 280: $17187-17195$.

Dubois RN, Abramson SB, Crofford L, Gupta RA, et al. (1998). Cyclooxygenase in biology and disease. FASEB J. 12: 1063-1073.

Fremont L (2000). Biological effects of resveratrol. Life Sci. 66: 663-673.

Gao Z and Ye J (2008). Inhibition of transcriptional activity of c-JUN by SIRT1. Biochem. Biophys. Res. Commun. 376: 793-796.

Holmes-McNary M and Baldwin AS Jr (2000). Chemopreventive properties of trans-resveratrol are associated with inhibition of activation of the IkappaB kinase. Cancer Res. 60: 3477-3483.

Howitz KT, Bitterman KJ, Cohen HY, Lamming DW, et al. (2003). Small molecule activators of sirtuins extend Saccharomyces cerevisiae lifespan. Nature 425: 191-196.

Jang M, Cai L, Udeani GO, Slowing KV, et al. (1997). Cancer chemopreventive activity of resveratrol, a natural product derived from grapes. Science 275: 218-220.

Jiang WJ (2008). Sirtuins: novel targets for metabolic disease in drug development. Biochem. Biophys. Res. Commun. 373: 341-344.

Juan ME, Vinardell MP and Planas JM (2002). The daily oral administration of high doses of trans-resveratrol to rats for 28 days is not harmful. J. Nutr. 132: 257-260.

Karin M (1999). How NF-kappaB is activated: the role of the IkappaB kinase (IKK) complex. Oncogene 18: 6867-6874.

Kotnik P, Nielsen J, Kwon TH, Krzisnik C, et al. (2005). Altered expression of COX-1, COX-2, and mPGES in rats with nephrogenic and central diabetes insipidus. Am. J. Physiol. Renal Physiol. 288: F1053-F1068.

Kumar A, Kaundal RK, Iyer S and Sharma SS (2007). Effects of resveratrol on nevre functions, oxidative stress and DNA fragmentation in experimental diabetic neuropathy. Life Sci. 80: 1236-1244.

Kundu JK and Surh YJ (2004). Molecular basis of chemoprevention by resveratrol: NF-kappaB and AP-1 as potential targets. Mutat. Res. 555: 65-80.

Lee JH, Song MY, Song EK, Kim EK, et al. (2009). Overexpression of SIRT1 protects pancreatic beta-cells against cytokine toxicity by suppressing the nuclear factor-kappaB signaling pathway. Diabetes 58 : 344-351.

Manna SK, Mukhopadhyay A and Aggarwal BB (2000). Resveratrol suppresses TNF-induced activation of nuclear transcription factors NF-kappa B, activator protein-1, and apoptosis: potential role of reactive oxygen intermediates and lipid peroxidation. J. Immunol. 164: 6509-6519.

Martinez J and Moreno JJ (2000). Effect of resveratrol, a natural polyphenolic compound, on reactive oxygen species and prostaglandin production. Biochem. Pharmacol. 59: 865-870.

Milne JC, Lambert PD, Schenk S, Carney DP, et al. (2007). Small molecule activators of SIRT1 as therapeutics for the 
treatment of type 2 diabetes. Nature 450: 712-716.

Nemoto S, Fergusson MM and Finkel T (2004). Nutrient availability regulates SIRT1 through a forkhead-dependent pathway. Science 306: 2105-2108.

Pervaiz S (2003). Resveratrol: from grapevines to mammalian biology. FASEB J. 17: 1975-1985.

Pfluger PT, Herranz D, Velasco-Miguel S, Serrano M, et al. (2008). Sirt1 protects against high-fat diet-induced metabolic damage. Proc. Natl. Acad. Sci. U. S. A. 105: 9793-9798.

Philip S, Bulbule A and Kundu GC (2001). Osteopontin stimulates tumor growth and activation of promatrix metalloproteinase-2 through nuclear factor-kappa B-mediated induction of membrane type 1 matrix metalloproteinase in murine melanoma cells. J. Biol. Chem. 276: 44926-44935.

Pillarisetti S (2008). A review of Sirt1 and Sirt1 modulators in cardiovascular and metabolic diseases. Recent Pat. Cardiovasc. Drug Discov. 3: 156-164.

Robertson RP and Harmon JS (2006). Diabetes, glucose toxicity, and oxidative stress: A case of double jeopardy for the pancreatic islet beta cell. Free Radic. Biol. Med. 41: 177-184.

Rodgers JT, Lerin C, Haas W, Gygi SP, et al. (2005). Nutrient control of glucose homeostasis through a complex of PGC1alpha and SIRT1. Nature 434: 113-118.

Sharma S, Anjaneyulu M, Kulkarni SK and Chopra K (2006). Resveratrol, a polyphenolic phytoalexin, attenuates diabetic nephropathy in rats. Pharmacology 76: 69-75.

Smith WL, DeWitt DL and Garavito RM (2000). Cyclooxygenases: structural, cellular, and molecular biology. Annu. Rev. Biochem. 69: 145-182.

St-Pierre Y, Couillard J and Van Themsche C (2004). Regulation of MMP-9 gene expression for the development of novel molecular targets against cancer and inflammatory diseases. Expert Opin. Ther. Targets 8: 473-489.

Stumvoll M, Goldstein BJ and van Haeften TW (2005). Type 2 diabetes: principles of pathogenesis and therapy. Lancet 365: 1333-1346.

Su HC, Hung LM and Chen JK (2006). Resveratrol, a red wine antioxidant, possesses an insulin-like effect in streptozotocininduced diabetic rats. Am. J. Physiol. Endocrinol. Metab. 290: E1339-E1346.

Subbaramaiah K, Chung WJ, Michaluart P, Telang N, et al. (1998). Resveratrol inhibits cyclooxygenase-2 transcription and activity in phorbol ester-treated human mammary epithelial cells. J. Biol. Chem. 273: 21875-21882.

Sulaiman M, Matta MJ, Sunderesan NR, Gupta MP, et al. (2010). Resveratrol, an activator of SIRT1, upregulates sarcoplasmic calcium ATPase and improves cardiac function in diabetic cardiomyopathy. Am. J. Physiol. Heart Circ. Physiol. 298: 833-843.

Surh YJ, Chun KS, Cha HH, Han SS, et al. (2001). Molecular mechanisms underlying chemopreventive activities of antiinflammatory phytochemicals: down-regulation of COX-2 and iNOS through suppression of NF-kappa B activation. Mutat. Res. 480-481: 243-268.

Szewczuk LM, Forti L, Stivala LA and Penning TM (2004). Resveratrol is a peroxidase-mediated inactivator of COX-1 but not COX-2: a mechanistic approach to the design of COX-1 selective agents. J. Biol. Chem. 279: 22727-22737.

Tanno M, Kuno A, Yano T, Miura T, et al. (2010). Induction of manganese superoxide dismutase by nuclear translocation and activation of SIRT1 promotes cell survival in chronic heart failure. J. Biol. Chem. 285: 8375-8382.

Tsai SH, Lin-Shiau SY and Lin JK (1999). Suppression of nitric oxide synthase and the down-regulation of the activation of NFkappaB in macrophages by resveratrol. Br. J. Pharmacol. 126: 673-680.

Woo JH, Lim JH, Kim YH, Suh SI, et al. (2004). Resveratrol inhibits phorbol myristate acetate-induced matrix metalloproteinase-9 expression by inhibiting JNK and PKC delta signal transduction. Oncogene 23: 1845-1853.

Wood JG, Rogina B, Lavu S, Howitz K, et al. (2004). Sirtuin activators mimic caloric restriction and delay ageing in metazoans. Nature 430: 686-689.

Yar AS, Menevse S, Alp E, Helvacioglu F, et al. (2010). The effects of resveratrol on cyclooxygenase-1 and cyclooxygenase-2 mRNA and protein levels in diabetic rat kidneys. Mol. Biol. Rep. 37: 2323-2331.

Yu C, Shin YG, Chow A, Li Y, et al. (2002). Human, rat, and mouse metabolism of resveratrol. Pharm. Res. 19: 19071914.

Yu H, Pan C, Zhao S, Wang Z, et al. (2008). Resveratrol inhibits tumor necrosis factor-alpha-mediated matrix metalloproteinase-9 expression and invasion of human hepatocellular carcinoma cells. Biomed. Pharmacother. 62: 366-372. 